\title{
Indian Hedgehog $b$ Function Is Required for the Specification of Oligodendrocyte Progenitor Cells in the Zebrafish CNS
}

\author{
Ah-Young Chung, ${ }^{1}$ Suhyun Kim, ${ }^{1}$ Eunmi Kim, ${ }^{1}$ Dohyun Kim, ${ }^{1}$ Inyoung Jeong, ${ }^{1}$ Young Ryun Cha, ${ }^{1}$ Young-ki Bae, ${ }^{2}$ \\ Seung Woo Park, ${ }^{3}$ Jehee Lee, ${ }^{4}$ and Hae-Chul Park ${ }^{1}$ \\ ${ }^{1}$ Graduate School of Medicine, Korea University, Ansan, Gyeonggido 425-707, Republic of Korea, ${ }^{2}$ Research Institute, National Cancer Center, Goyang, \\ Gyeonggido 410-769, Republic of Korea, ${ }^{3}$ Department of Internal Medicine, Brain Korea 21 Project, Severance Hospital, Yonsei University College of \\ Medicine, Seoul 120-752, Republic of Korea, and ${ }^{4}$ Department of Marine Life Sciences, School of Marine Biomedical Sciences, Jeju National University, Jeju \\ Self-Governing Province 690-756, Republic of Korea
}

A subset of ventral spinal cord precursors, known as pMN precursor cells, initially generate motor neurons and then oligodendrocyte progenitor cells (OPCs), which migrate and differentiate as myelinating oligodendrocytes in the developing neural tube. The switch between motor neuron and oligodendrocyte production by the pMN neural precursors is an important step in building a functional nervous system. However, the precise mechanism that orchestrates the sequential generation of motor neurons and oligodendrocytes within the common population of pMN precursors is still unclear. The current study demonstrates that Indian Hedgehog b (Ihhb), previously known as Echidna Hedgehog, begins to be expressed in the floor plate cells of the ventral spinal cord at the time of OPC specification in zebrafish embryos. Ihhb loss-of-function analysis revealed that Ihhb function is required for OPC specification from $\mathrm{pMN}$ precursors by negatively regulating the proliferation of neural precursors. Finally, results showed that Sonic Hedgehog (Shh) could not replace Ihhb function in OPC specification, suggesting that Ihhb and Shh play separate roles in OPC specification. Altogether, data from the present study suggested a novel mechanism, mediated by Ihhb, for the sequential generation of motor neurons and oligodendrocytes from pMN precursors in the ventral spinal cord of zebrafish embryos.

\section{Introduction}

During vertebrate neural development, proliferative neuroepithelial precursors give rise first to neurons and later to glial cells. A particularly good example of this process is seen with a specific subset of ventral spinal cord precursor cells known as pMN precursors, which produce motor neurons during neurogenesis. The production of motor neurons is followed by the generation of oligodendrocyte progenitor cells (OPCs), which form oligodendrocytes, the myelinating cell type of the CNS (Rowitch, 2004). However, the precise mechanism that orchestrates the sequential generation of motor neurons and oligodendrocytes within the common population of pMN precursors is still unclear.

In the developing spinal cord, morphogenetic gradient of Sonic hedgehog (Shh) induces distinct pMN precursor domains

\footnotetext{
Received July 16, 2012; revised Nov. 6, 2012; accepted Nov. 30, 2012.

Author contributions: A.-Y.C. and H.-C.P. designed research; A.-Y.C., S.K., E.K., D.K., and I.J. performed research; S.W.P. and J.L. contributed unpublished reagents/analytic tools; A.-Y.C., S.K., Y.R.C., Y.-k.B., J.L., and H.-C.P. analyzed data; A.-Y.C. and H.-C.P. wrote the paper.

This research was supported by the Brain Research Program (2011-0019233) and Basic Science Research Program (2009-0075120) through the National Research Foundation of Korea (NRF) funded by the Ministry of Education, Science and Technology.

The authors declare no competing financial interests.

Correspondence should be addressed to either of the following: Hae-Chul Park, Graduate School of Medicine, Korea University, Ansan, Gyeonggido 425-707, Republic of Korea; or Jehee Lee, Marine Molecular Genetics Laboratory, Department of Marine Life Sciences, College of Ocean Science, Jeju National University, 66 Jejudaehakno, Ara-Dong, Jeju 690-756, Republic of Korea. E-mail: hcpark67@korea.ac.kr or jehee@jejunu.ac.kr.

DOI:10.1523/JNEUROSCI.3369-12.2013

Copyright $@ 2013$ the authors $\quad 0270-6474 / 13 / 331728-06 \$ 15.00 / 0$
}

in the ventral spinal cord, and then Shh signaling is required for the generation of motor neuron and OPCs from the pMN precursors (Poncet et al., 1996; Pringle et al., 1996; Lewis and Eisen, 2001; Park et al., 2002). However, it is not clear how Hh signaling instructs the complex sequence of motor neurons and OPC specification that occurs over time during development. The present study shows that Indian Hedgehog b (Ihhb, previously known as Echidna Hedgehog), another member of the Hh signaling pathway, begins to be expressed in the floor plate cells of the ventral spinal cord at the time of OPC specification in zebrafish embryos. An Ihhb loss-of-function study revealed that in the absence of Ihhb function, neural precursors failed to specify OPCs and instead proliferated continuously to generate neurons, indicating that Ihhb function is required for the specification of OPCs from pMN precursors by negatively regulating the proliferation of neural precursors. Finally, we found that Shh could not replace Ihhb function in OPC specification, suggesting that Ihhb and Shh play separate roles in OPC specification. Together, our data suggested a novel mechanism, mediated by Ihhb, for the sequential generation of motor neurons and oligodendrocytes from pMN precursors in the ventral spinal cord of zebrafish embryos.

\section{Materials and Methods}

Fish lines and cyclopamine treatment. Wild-type AB, $\operatorname{Tg}$ (olig2:egfp) (Shin et al., 2003), $\operatorname{Tg}$ (olig2:dsred) (Kucenas et al., 2008), $\operatorname{Tg}$ (hsp70:gal4) (Scheer and Campos-Ortega, 1999), $T g$ (uas:shha) (Jung et al., 2011), and $\mathrm{Tg}$ (hsp70:ihhb:mCherry) zebrafish of either sex were used for this study. 

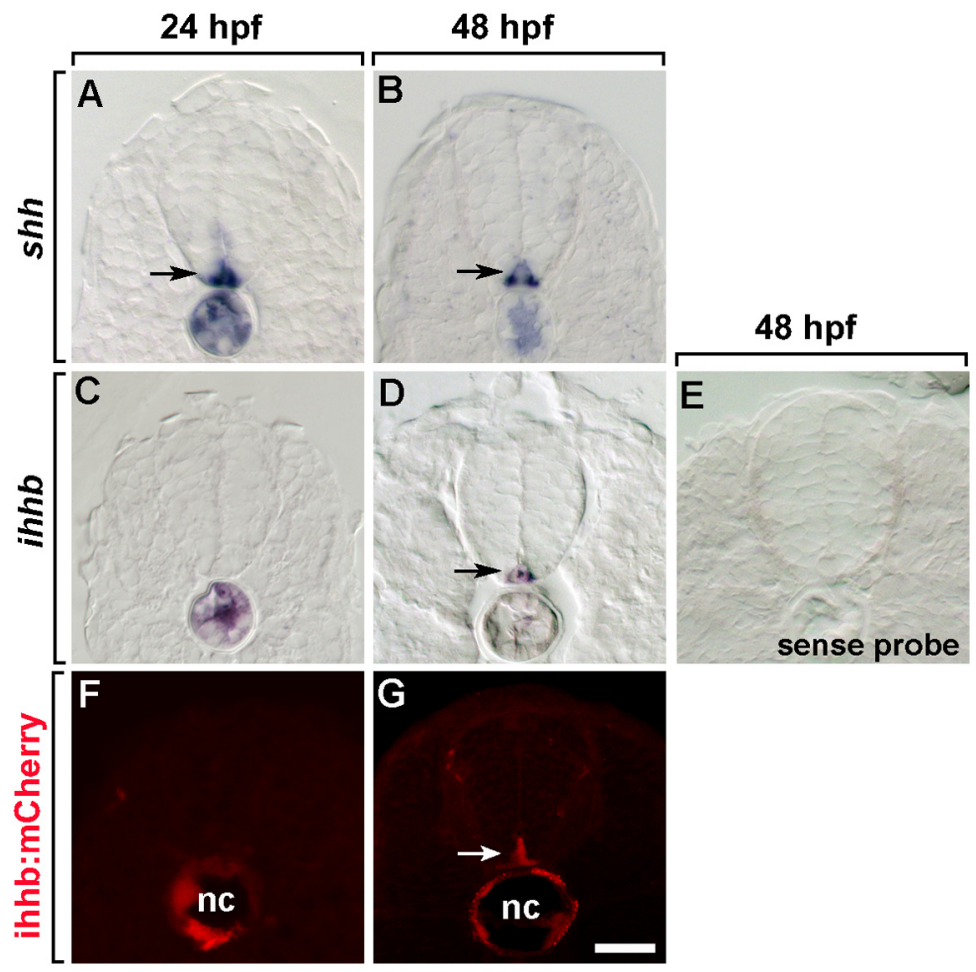

Figure 1. Ihhb begins to be expressed in the spinal cord at the time when $O P C$ s are specified. $A-G$, Transverse sections of spinal cord, dorsal to the top. $\boldsymbol{A}-\boldsymbol{E}$, RNA in situ hybridization with $\operatorname{sh}(\boldsymbol{A}, \boldsymbol{B})$ and ihhb antisense probes $(\boldsymbol{C}, \boldsymbol{D})$, and ihhb sense probes $(\boldsymbol{E})$ in wild-type embryos. $\boldsymbol{F}, \mathbf{G}, \mathrm{mCherry}$ expression in the notochord and floor plate cells of ihhb:mCherry DNA-injected embryos. Arrows indicate floor plate cells. nc, Notochord. Scale bar, $20 \mu \mathrm{m}$.

For cyclopamine treatment, embryos were incubated in embryo medium (EM) containing $100 \mu \mathrm{M}$ cyclopamine (Toronto Research Chemicals) diluted from a $10 \mathrm{~mm}$ stock in ethanol at $28.5^{\circ} \mathrm{C}$.

Plasmid construction and bacterial artificial chromosome homologous recombination. To produce $\mathrm{Tg}$ (hsp70:ihhb:mCherry) fish, PCR was used to amplify ihhb using a forward primer containing an attB1 site (5'-GG GGACAAGTTTGTACAAAAAAGCAGGCTTTATGAGACTCTCCACG GCG- $\left.3^{\prime}\right)$ and a reverse primer containing an attB2 site (5'-GGGGACCA CTTTGTACAAGAAAGCTGGGTTGGATCTCTCAGTTGCCTC-3'). The PCR product containing the attB sites was cloned into a middleentry vector using the BP reaction of the Gateway system (Invitrogen). A $5^{\prime}$ entry clone containing a fragment of the zebrafish heat shock 70 ( $h s p 70$ ) promoter and a $3^{\prime}$ entry clone containing the mcherry were kindly provided by Chi-Bin Chien, University of Utah, Salt Lake City, UT (Kwan et al., 2007). The Gateway LR reactions were performed using LR II clonase with the entry clones, according to the manufacturer's recommendations (Invitrogen).

The bacterial artificial chromosome (BAC) clone for ihhb (CH211237C6) was purchased from GenomeCube to generate ihhb:mCherry Bac DNA. Targeting vectors were made by multisite Gateway cloning system (Invitrogen). The $5^{\prime}$ entry vector was generated by cloning of PCR product containing $750 \mathrm{bp}$ of left-side homologous arms for ihhb into p5EMCS vector (Kwan et al., 2007) linearized with SmaI. A middle entry clone was generated by inserting PCR product including mcherry sequence, FRT-Kan-FRT cassette, and I-SceI recognition site. A 3' entry vector contains $700 \mathrm{bp}$ of right-side homologous arms of the ihhb coding region. LR reaction was performed to generate final targeting vector using LR II clonase with the three entry clones. Targeting vector was linearized by KpnI/BglI digestion and electroporated into EL250 cells that harbor ihhb BAC clone. The BAC homologous recombination was performed as previously described (Shin et al., 2003).

Bromodeoxyuridine labeling, immunohistochemistry, and in situ RNA hybridization. Embryos were labeled with bromodeoxyuridine (BrdU; Roche) by incubating them for $20 \mathrm{~min}$ on ice in a solution of $10 \mathrm{~mm} \mathrm{BrdU}$ and $15 \%$ dimethyl sulfoxide in EM. The embryos were then placed in
$\mathrm{EM}$, incubated for $20 \mathrm{~min}$ at $28.5^{\circ} \mathrm{C}$, and fixed using $4 \%$ paraformaldehyde. Embryos were processed for immunohistochemistry, treated for $1 \mathrm{~h}$ with $2 \mathrm{M} \mathrm{HCl}$, and then processed for anti-BrdU immunohistochemistry. For immunohistochemistry, we used the following primary antibodies: rabbit anti-Sox10 (Park et al., 2005), rabbit anti-GFP (1:500, Abcam), mouse anti-BrdU (G3G4, 1:1000, Developmental Studies Hybridoma Bank), mouse antiIsl (39.4D5, 1:100, Developmental Studies Hybridoma Bank), and mouse anti-HuC/D (16A11 1:20, Invitrogen). Alexa Fluor 488- and 568 -conjugated secondary antibodies were used for fluorescence detection (1:500, Invitrogen). In situ RNA hybridization was performed with previously described RNA probes including ihhb (Currie and Ingham, 1996), shh (Krauss et al., 1993), olig2 (Park et al., 2002), and ngn1 (Blader et al., 1997).

Morpholino and RNA injection. A previously described morpholino oligonucleotide (MO) for $i h h b$ (ihhb MO, 5'-ATGAGACTCT CCACGGCGGCGGCGCTCCTC-3') (Lewis and Eisen, 2001) and scrambled MO (5' -CCTC TTACCTCAGTTACAATTTATA-3') were purchased from Gene Tools, LLC. The MOs were dissolved in $1 \times$ Danieau solution at a concentration of $20 \mu \mathrm{g} / \mu \mathrm{l}$ and diluted further with distilled water, and then $5 \mathrm{ng}$ of MOs were injected into one-cell stage zebrafish embryos. For RNA injection, 50 pg of olig2 RNA was injected into one-cell embryos.

Fluorescence-activated cell sorting. Approximately 300 wild-type and $i h h b \mathrm{MO}$-injected 2 days postfertilization (dpf) $\mathrm{Tg}$ (olig2:egfp) embryos were used to isolate $\mathrm{GFP}^{+}$cells by fluorescenceactivated cell sorting (FACS). Cell dissociation and FACS were performed as previously described (Chung et al., 2011) using a FACSAriaII (Becton Dickinson).

$R N A$ extraction and quantitative reverse transcription-PCR. Embryos were collected and homogenized in TRIzol solution (Invitrogen) to purify total RNA. Real-time quantitative reverse transcription PCR (qRT-PCR) was performed using a LightCycler (Roche) in a reaction mixture containing $1 \mu \mathrm{l}$ of $\mathrm{PCR}$-amplified total $\mathrm{CDNA}$ as a template, $0.2 \mathrm{M} 5^{\prime}$ and 3' PCR primers, $0.8 \mu \mathrm{l}$ of $\mathrm{MgCl}_{2}$, and $1 \mu \mathrm{l}$ of LightCycler Fast Start DNA Master SYBR Green I (Roche). The following oligonucleotide primers were used for qRT-PCR: $c d k n 1 c$ forward (F) (5' -CAAGAATCCGAGGGAGTCCC-3'); reverse (R) (5' -GT TCATCCTGCTTCGACTCC-3'); cyclinEF ( $5^{\prime}$ - AGGCTCTCGACATT CAAGGA-3'); R (5'-TCGAGTCTTATGCCAATGCTT-3'); $c d k 2$ F (5'-GGCCTGGAGTTACATCAATGC-3'); R (5'-CCCGTCTTGCCA AAGTTGATCT- $\left.3^{\prime}\right) ; \beta$-actin $\mathrm{F}\left(5^{\prime}\right.$-TAGTCATTCCAGAAGCGTTTAC C-3'); and R (5' -TACAGAGACACCCTGGCTTACAT-3').

\section{Results}

Ihhb signaling is required for the specification of OPCs in the ventral spinal cord

In previous studies, we have shown that the zebrafish Hh-related genes, shh and tiggy-winkle hedgehog ( $t w h h)$, are expressed in the floor plate cells of the ventral spinal cord, and that their function is required for the induction and maintenance of olig2 expression, which is crucial for motor neuron and oligodendrocyte development in the pMN precursor domain of the spinal cord (Park et al., 2004). We also have shown previously that overexpression of olig2 failed to rescue defects in OPC specification in embryos treated with cyclopamine, a specific inhibitor of the Hedgehog signaling pathway (Chen et al., 2002), suggesting that Hh signal- 

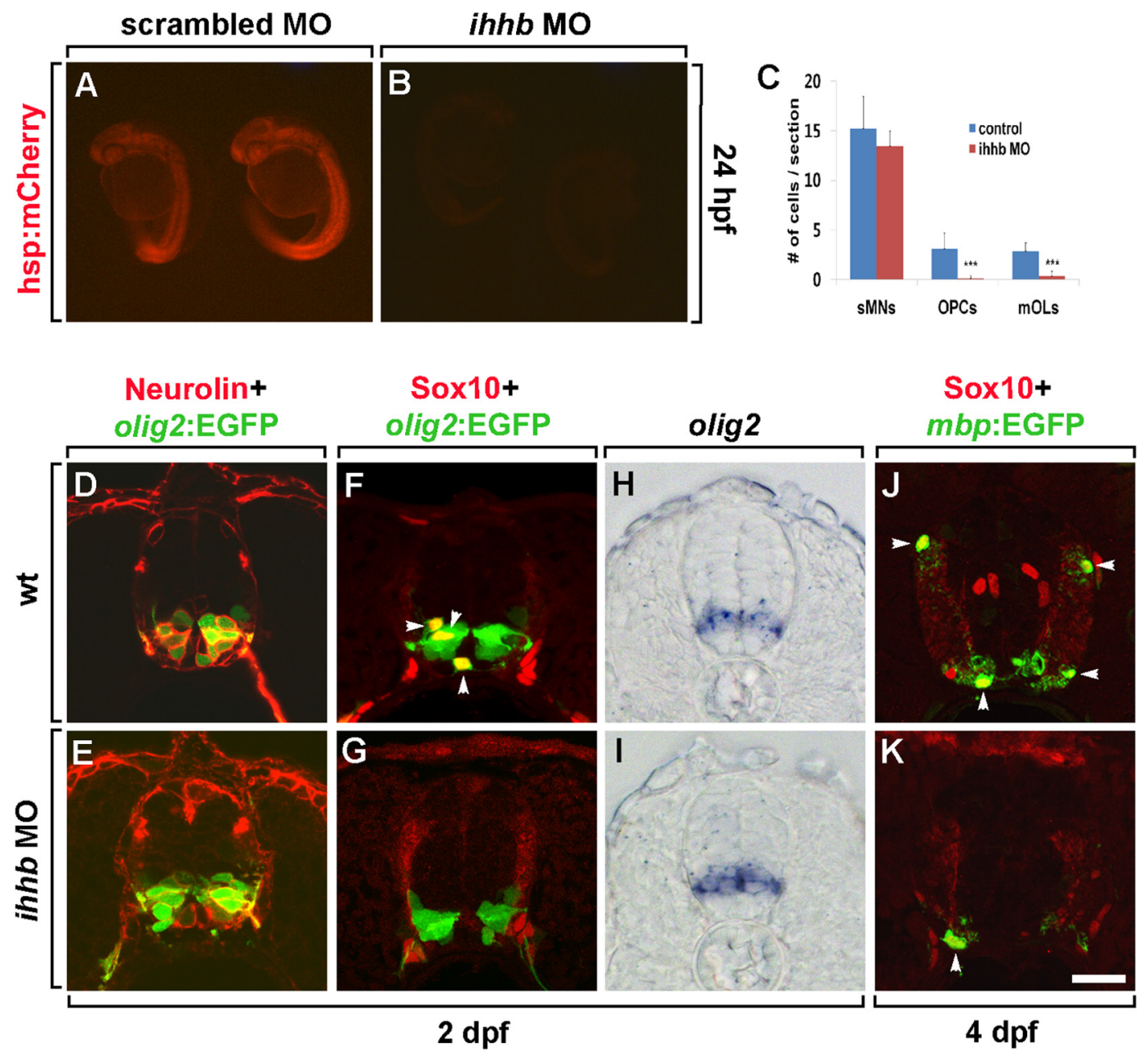

Figure 2. Ihhb function is required for the specification of OPCS. $\boldsymbol{A}, \boldsymbol{B}$, Scrambled M0-injected control ( $\boldsymbol{A}$ ) and ihhb M0-injected (B) $\operatorname{Tg}($ hsp 70 :ihhb-mcherry) embryos heat shocked at 20 hpf. $\boldsymbol{C}$, Quantification of motor neurons (sMNs), OPCs, and mature oligodendrocytes ( $\mathrm{mOLs}$ ) in wild-type and ihhb M0-injected embryos $\left.*^{* * *} p<0.001\right)$. Data were obtained from 15 sections from each of 6 control and 6 ihhb M0-injected embryos. $\boldsymbol{D}-\boldsymbol{K}$, Transverse sections of spinal cord, dorsal to the top. Wild-type $(\boldsymbol{D}, \boldsymbol{F})$ and ihhb M0-injected $(\boldsymbol{E}, \boldsymbol{G}) \operatorname{Tg}($ oligz:egfp) embryos were labeled with anti-Neurolin $(\boldsymbol{D}, \boldsymbol{E})$ and anti-Sox10 $(\boldsymbol{F}, \boldsymbol{G})$ antibodies. $\boldsymbol{H}, \boldsymbol{I}, \mathbf{I n}$ situ RNA hybridization of wild-type $(\boldsymbol{H})$ and ihhb M0-injected $(\boldsymbol{I})$ embryos with an olig2 probe. $\boldsymbol{J}, \boldsymbol{K}$, Wild-type $(\boldsymbol{J})$ and ihhb M0-injected $(\boldsymbol{K})$ $T g$ (mbp:egfp) embryos were labeled with anti-Sox 10 antibody. Arrowheads indicate oligz:EGFP ${ }^{+} / \mathrm{Sox} 10^{+} \mathrm{OPCs}$ in $\boldsymbol{F}$ and $\mathrm{mbp}: \mathrm{EGFP}^{+} / \mathrm{Sox} 10^{+}$mature oligodendrocytes in $J$ and $\boldsymbol{K}$. Scale bar, $20 \mu \mathrm{m}$.

ing might play an additional role in OPC specification in parallel with the maintenance of olig2 expression in the pMN precursor domain (Park et al., 2002). In contrast to shh and twhh, ihhb has been shown to be expressed exclusively in the notochord and to be required for the formation of muscle pioneer cells in developing somites (Currie and Ingham, 1996).

To explore the function of $\mathrm{Hh}$ signaling in OPC specification, we first investigated the expression of shh and Ihhb in the developing spinal cord using in situ RNA hybridization. As shown in Figure 1, shh was expressed in the notochord and floor plate cells throughout neurogenesis [24 hours postfertilization (hpf)] and oligodendrogenesis ( $48 \mathrm{hpf}$ ), although its expression decreased at $48 \mathrm{hpf}$ (Fig. $1 A, B$ ). Ihhb was expressed exclusively in notochord cells and was not detected in the spinal cord at $24 \mathrm{hpf}$; however, floor plate cells began to express Ihhb at $36 \mathrm{hpf}$ (data not shown) and this expression was clearly detectable in floor plate cells and notochord cells at $48 \mathrm{hpf}$ (Fig. 1C,D) but was not detectable with a sense probe for ihhb (Fig. 1E). To confirm Ihhb expression in the spinal cord, an ihhb:mcherry BAC DNA, which expresses mCherry under the control of the $i h h b$ promoter, was generated using Escherichia coli-based homologous recombination (Shin et al., 2003). Consistent with the in situ RNA hybridization data, embryos injected with ihhb:mcherry BAC DNA expressed
mCherry in the notochord only at $24 \mathrm{hpf}$ but in the notochord and floor plate cells at $48 \mathrm{hpf}$ (Fig. $1 F, G$ ), suggesting that Ihhb might play a role in OPC specification.

To investigate Ihhb function in oligodendrocyte development, we used an antisense MO against ihhb (ihhb MO), which was previously reported to block ihhb expression (Lewis and Eisen, 2001). The specificity of the ihhb MO was further confirmed by injecting it into $\mathrm{Tg}$ (hsp70:ihhb-mcherry) zebrafish embryos, which expressed an mCherry-tagged Ihhb protein by heat shock 70 promoter. When the embryos were heat shocked at $39^{\circ} \mathrm{C}$ for $30 \mathrm{~min}$, a very low level of mCherry expression was detected (Fig. 2B), whereas scrambled MO-injected control embryos displayed robust mCherry expression (Fig. $2 A$ ), demonstrating that the ihhb MO inhibited ihhb expression efficiently.

Next, Ihhb loss-of-function was tested by injecting the ihhb MO into $T g$ (olig2:egfp) embryos. Since ihhb is first expressed in the spinal cord at $36 \mathrm{hpf}$, when secondary motor neurons (sMNs) are already specified, embryos injected with the $i h h b \mathrm{MO}$ showed normal development of $\mathrm{EGFP}^{+} / \mathrm{Neurolin}^{+}$sMNs (Fig. 2C-E). However, anti-Sox 10 antibody labeling of the spinal cord section revealed that $i h h b$ MO-injected $\operatorname{Tg}$ (olig2:egf $p$ ) embryos failed to specify $\mathrm{EGFP}^{+} / \mathrm{Sox}_{10} 0^{+} \mathrm{OPCs}$ at $2 \mathrm{dpf}$ (Fig. $2 C, F, G$ ), indicating that Ihhb function is required for OPC specification. Interest- 

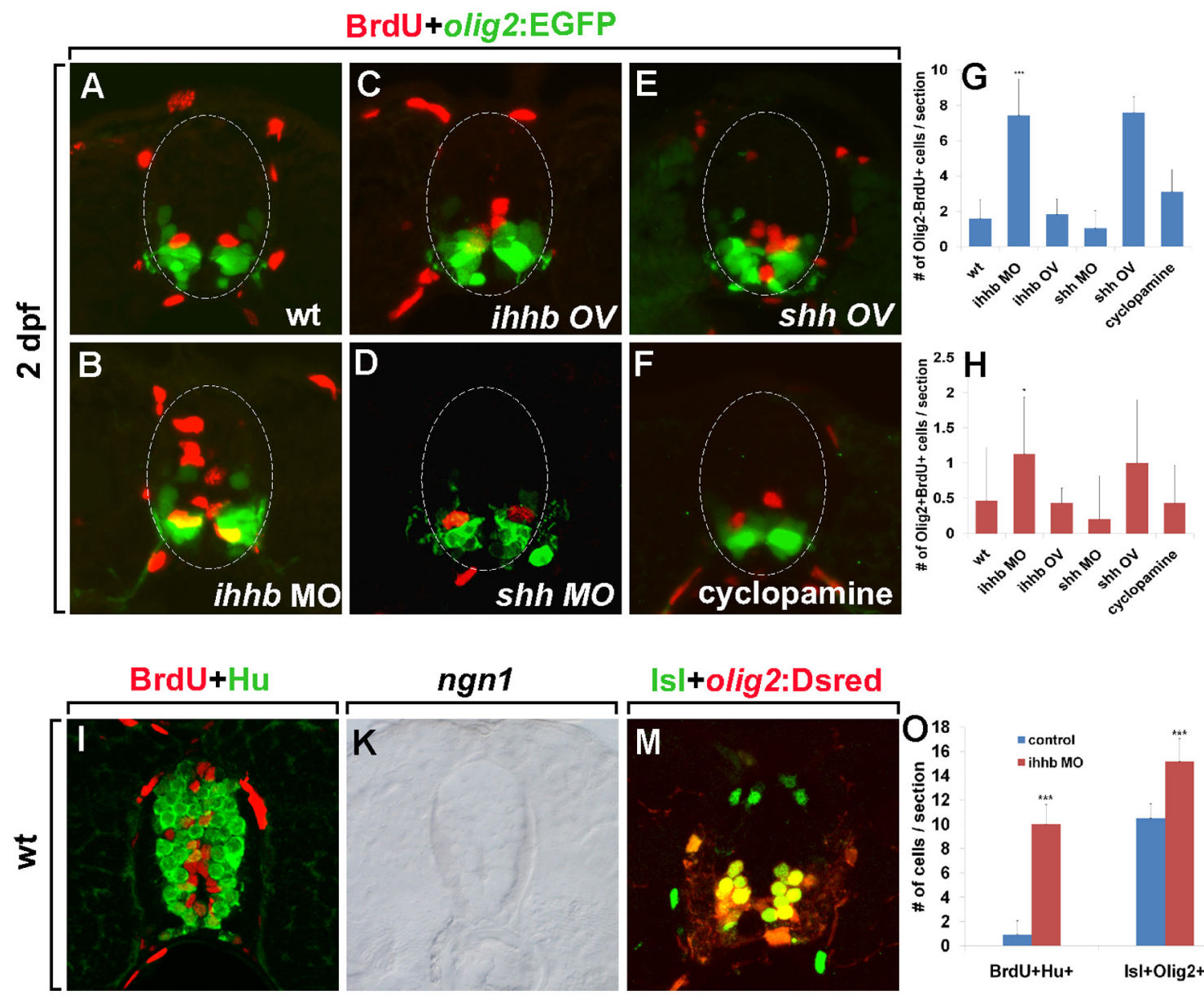

Is\|+olig2:Dsred

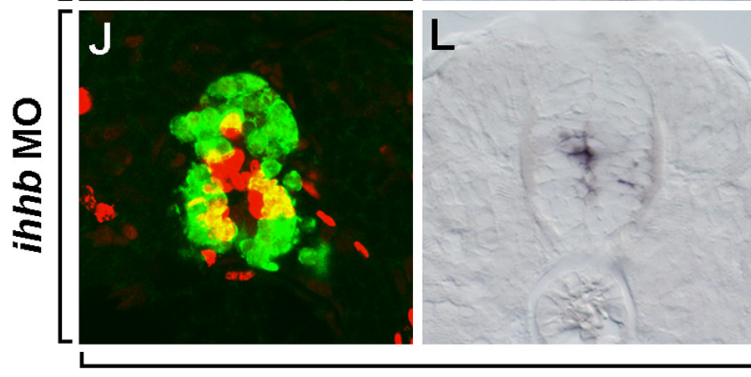

$3 \mathrm{dpf}$
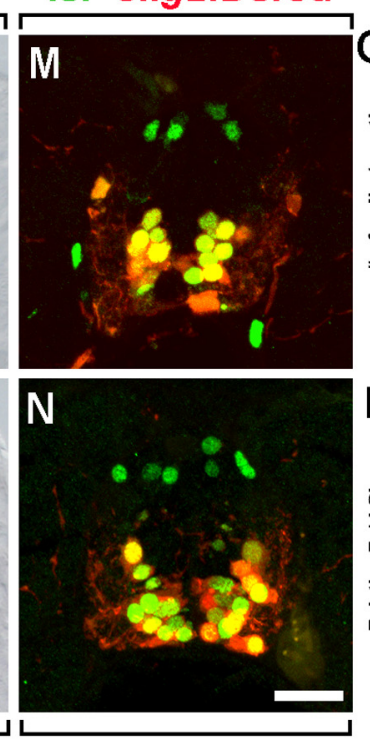

$4 \mathrm{dpf}$

Figure 3. Inhb function is required for neurogenesis-oligodendrogenesis switching by regulating precursor proliferation. $\boldsymbol{A}-\boldsymbol{F}$, Transverse sections of the spinal cord of $T g(o l i g 2: e g f p)$ embryos,

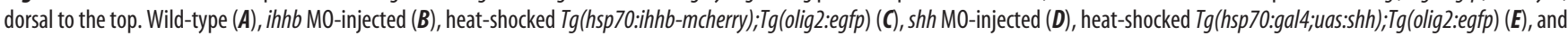
cyclopamine-treated (F) Tg(oligz:egfp) embryos were treated with BrdU for $20 \mathrm{~min}$ at $2 \mathrm{dpf}$, and labeled with anti-BrdU antibody. Dashed circles outline the spinal cord. $\mathbf{G}, \boldsymbol{H}, \mathbf{Q u a n t i f i c a t i o n ~ o f ~}$ $\mathrm{BrdU}^{+} / 0$ lig2:EGFP ${ }^{-}$cells $\left({ }^{* * *} p<0.001\right)(\boldsymbol{G})$ and BrdU ${ }^{+} / 0$ lig2:EGFP ${ }^{+}$cells $\left({ }^{*} p<0.05\right)$. OV, Overexpression. Data were obtained from 15 sections from each of 6 control and 6 M0-injected embryos. I-N, Transverse sections of the spinal cord, dorsal to the top. $(\boldsymbol{I}, \boldsymbol{J})$ Wild-type $(\boldsymbol{I})$ and $i h h b \mathrm{MO}$-injected $(\boldsymbol{J})$ embryos were incubated with BrdU for 20 min at $2 \mathrm{dpf}$, cultured for one more day, and then labeled with anti-BrdU and anti-Hu antibodies at $3 \mathrm{dpf} . \boldsymbol{K}, \boldsymbol{L}, \mathbf{I n}$ situ RNA hybridization of wild-type $(\boldsymbol{K})$ and $i h h b$ M0-injected $(\boldsymbol{L})$ embryos with a ngn 1 probe. $\boldsymbol{M}, \boldsymbol{N}, \mathbf{L a b e l i n g}$ of wild-type $(\boldsymbol{M})$ and ihhb M0-injected $(\boldsymbol{N}) \mathrm{Tg}$ (oligz:dsred) embryos with anti-Isl antibody at 4 dpf. $\mathbf{0}$, Quantification of BrdU ${ }^{+} / \mathrm{Hu}^{+}$cells from I and $\boldsymbol{J}$, and Isl ${ }^{+} /$oligz:Dsred ${ }^{+}$cells from $\boldsymbol{M}$ and $\boldsymbol{N}$. Data were obtained from 15 sections from each of 6 control and 6 ihhb M0-injected embryos. $P$, Real-time RT-PCR analysis to compare the expression of cell cycle regulators in wild-type, ihhb M0-injected embryos and oligz:EGFP ${ }^{+}$cells FACS-isolated from wild-type and ihhb M0-injected Tg(oligz:egfp) embryos. Data are shown as the average fold change relative to the wild-type control and are normalized to $\beta$-actin expression. The results are presented as the mean \pm SD from three independent experiments ( $\left.{ }^{* * *} p<0.001\right)$. Scale bar, $20 \mu \mathrm{m}$.

ingly, ihhb MO-injected embryos showed normal levels of olig2 expression, as revealed by in situ RNA hybridization with olig2 (Fig. 2I), indicating that in contrast to Shh, Ihhb is not required for the maintenance of olig2 expression. Consistent with loss of Sox $10^{+}$OPCs, ihhb MO-injected $\operatorname{Tg}(m b p: E G F P)$ embryos, which express EGFP in mature oligodendrocytes (Jung et al., 2010), show a dramatically reduced number of Sox $10^{+} / \mathrm{mbp}$ : $\mathrm{EGFP}^{+}$mature oligodendrocytes (Fig. 2C,J,K).
Ihhb function is required for neurogenesisoligodendrogenesis switching by regulating precursor proliferation

A previous study has shown that $i h h$ is required for the cell cycle exit and differentiation of colonic epithelial cells in the mammalian gut (van den Brink et al., 2004), suggesting that Ihhb function might be involved in the regulation of neural precursor proliferation during oligodendrogenesis. To test this idea, we used thy- 
midine analog BrdU. The spinal cord of control $\mathrm{Tg}$ (olig2:egfp) embryos exhibited many BrdU ${ }^{+}$proliferating neural precursors during neurogenesis at $24 \mathrm{hpf}$ (data not shown) but showed few $\mathrm{BrdU}^{+}$proliferating neural precursors during oligodendrogenesis at $2 \mathrm{dpf}$ (Fig. 3A). Interestingly, $\operatorname{Tg}$ (olig2:egfp) embryos injected with ihhb MO exhibited a number of proliferating cells similar to that of wild-type embryos at $24 \mathrm{hpf}$ (data not shown) but showed a dramatic increase in the number of both $\mathrm{BrdU}^{+} /$olig2:EGFP ${ }^{+}$ and $\mathrm{BrdU}^{+} /$olig2: $\mathrm{EGFP}^{-}$cells at $2 \mathrm{dpf}$, after $i h h b$ expression begins in the spinal cord (Fig. $3 B, G, H$ ). These results indicate that inhibition of Ihhb function caused defects in cell cycle regulation, which resulted in increased proliferation of neural precursors throughout the spinal cord.

qRT-PCR revealed that the expression of cell cycle components, including $c d k 2$ and $c y c l i n E$, was increased, while the expression of cyclin-dependent kinase inhibitor lc $(c d k n 1 c)$, a cell cycle inhibitor for neural precursors in the zebrafish CNS (Park et al., 2005), was decreased throughout the spinal cord and in FACS-purified olig2:EGFP ${ }^{+}$ cells from $i h h b \mathrm{MO}$-injected embryos at $2 \mathrm{dpf}$ (Fig. 3P). The spinal cords of $\operatorname{Tg}$ (hsp70:ihhb-mcherry); $\operatorname{Tg}$ (olig2:egfp) embryos showed a similar amount of proliferation of neural precursors after heat shock induction of Ihhb (Fig. $3 C, G, H$ ), indicating that overexpression of Ihhb alone does not induce cell cycle arrest in the remaining neural precursors at $2 \mathrm{dpf}$. Interestingly, the number of $\mathrm{BrdU}^{+}$proliferating neural precursors in the spinal cords of shh MO-injected embryos was decreased (Fig. 3D, G,H), but overexpression of Shh in heat-shocked $\mathrm{Tg}$ (hsp70:gal4;uas:shh); $\mathrm{Tg}$ (olig2:egfp) embryos caused a dramatic increase in $\mathrm{BrdU}^{+}$proliferating precursors throughout the spinal cord at $2 \mathrm{dpf}$ (Fig. $3 E, G, H)$. This suggests that in contrast to Ihhb, Shh has a stimulatory effect on cell cycle progression during oligodendrogenesis. Embryos treated with cyclopamine showed a similar number of proliferating neural precursors to wild-type embryos at $2 \mathrm{dpf}$ (Fig. 3F-H).

Since the data described above demonstrated that neural precursor proliferation increased without OPC specification in the Ihhb-deficient spinal cord, next we performed a BrdU pulsechase experiment to test whether proliferating precursors generated neurons instead of OPCs in the absence of Ihhb function during oligodendrogenesis. After the incubation of wild-type and ihhb MO-injected embryos with BrdU for $20 \mathrm{~min}$ at $2 \mathrm{dpf}$ during oligodendrogenesis, the embryos were cultured for one more day and then labeled with an anti-BrdU antibody together with anti-Hu antibody to label postmitotic neurons. In wild-type embryos, few $\mathrm{BrdU}^{+} / \mathrm{Hu}^{+}$neurons were observed (Fig. 3I,O), while ihhb MO-injected embryos show an increased number of $\mathrm{BrdU}^{+} / \mathrm{Hu}^{+}$postmitotic neurons in the spinal cord at $3 \mathrm{dpf}$ (Fig. $3 \mathrm{~J}, \mathrm{O}$ ), indicating that in the absence of Ihhb function, proliferating spinal precursors continuously generated neurons during oligodendrogenesis. Consistent with this, the expression of ngn1, a basic-helix-loop-helix transcription factor that is required for neurogenesis, was maintained at significant levels in the spinal cords of ihhb MO-injected embryos (Fig. 3L)
Sox10

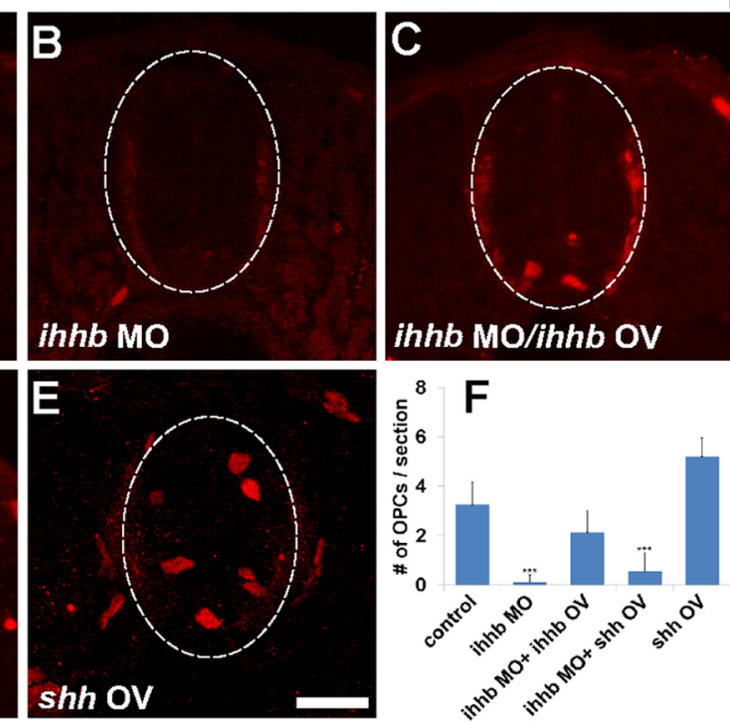

but was downregulated in wild-type embryos at $3 \mathrm{dpf}$ (Fig. $3 K$ ). As a consequence of continuous neurogenesis, an increased number of $\mathrm{Isl}^{+} /$olig2:Dsred ${ }^{+}$motor neurons was observed at $4 \mathrm{dpf}$ (Fig. $3 \mathrm{M}, \mathrm{O}$ ) in the spinal cords of ihhb MO-injected Tg(olig2: Dsred) embryos. Together, these data suggest that Ihhb regulation of neural precursor proliferation is required for the switch from neurogenesis to oligodendrogenesis.

Since our data suggested that Shh and Ihhb appear to play different roles in cell cycle regulation and olig2 induction in neural precursors during oligodendrogenesis, we tested whether impaired OPC specification due to ihhb MO injection could be recovered by overexpression of Ihhb and Shh. First, ihhb expression was induced at a high level by heat shocking $\operatorname{Tg}(h s p 70$ :ihhbmcherry) embryos twice at $24 \mathrm{hpf}$ and $36 \mathrm{hpf}$ after injection of the ihhb MO, followed by embryo analysis. Although the data described above showed that the ihhb MO could interfere with transgenic ihhb:mcherry expression during early development, we reasoned that sufficiently high expression of the transgene should produce transcripts in excess of the $i h h b \mathrm{MO}$ as the MO becomes diluted during development. As expected, these embryos partially rescued Sox $10^{+}$OPC production (Fig. 4C,F) compared with ihhb MO-injected transgenic embryos without heat shock (Fig. 4B). However, in contrast to the increased number of Sox $10^{+}$OPCs in noninjected $\mathrm{Tg}$ (hsp70:gal4;uas:shh) embryos (Fig. $4 E, F$ ), transgenic embryos injected with the ihhb MO and heat-shocked twice at $24 \mathrm{hpf}$ and $36 \mathrm{hpf}$ failed to rescue OPC production (Fig. $4 D, F$ ), indicating that Shh could not compensate for the loss of Ihhb function in OPC specification. Together, these data suggested that Ihhb and Shh play different roles in OPC specification.

\section{Discussion}

In the present study, we reported that $i h h b$ expression begins in the floor plate cells of the ventral spinal cord at the time when OPCs are specified, and that Ihhb function is required for OPC specification. Ihhb negatively regulates the proliferation of spinal 
precursors, partly by regulating the expression of $c d k n 1 c$, a negative regulator of the cell cycle. These data suggested a novel mechanism, mediated by Ihhb, for the sequential generation of motor neurons and oligodendrocytes from pMN precursors in the ventral spinal cord. Interestingly, loss-of-Ihhb function caused increased proliferation of neural precursors throughout the spinal cord, indicating that the effect of Ihhb is not restricted to the olig2-expressing pMN domain but is a general effect on the cell cycle in spinal cord precursors. These data suggest the possibility that Ihhb function is required for the generation of glial cells throughout the spinal cord, including oligodendrocytes and astrocytes; however, we could not confirm this hypothesis because there is no available marker specific for astrocytes in zebrafish.

Recently, it has been shown that the phosphorylation state of olig2 regulates the proliferation of neural progenitors in the developing forebrain (Li et al., 2011) and the motor neuronoligodendrocyte fate switch in the developing spinal cord (Sun et al., 2011). In the developing spinal cord, phosphorylation of Olig2 is required for motor neuron specification, but subsequent dephosphorylation of Olig2 triggers oligodendrocyte fate specification (Li et al., 2011). Since our results show that ihhb function is required for the motor neuron-oligodendrocyte fate switch, these studies suggest the possibility that Ihhb function might be involved in regulation of the phosphorylation state of Olig2 or in regulation of cofactor expression, which is required to activate oligodendrocyte specification and repress motor neuron development.

Our data also suggested that both Shh and Ihhb are required for OPC specification but that they have separate functions. Ihhb is required for the cell cycle inhibition of the spinal precursors involved in OPC specification, while Shh has a stimulatory effect on the cell cycle progression and maintenance of olig2 expression. These data conflict with previous studies, which have shown that Shh signaling from grafted notochord or floor plate promotes the generation of pMN-derived OPCs in the spinal cord (Poncet et al., 1996; Pringle et al., 1996). One possible explanation is that grafted notochord and floor plate cells can generate both Shh and Ihh, and could thus contribute to generate oligodendrocyte lineage cells together. A similar mechanism for the regulation of proliferation versus differentiation involving separate roles for Shh and Ihh also has been shown in the regulation of colonic epithelium differentiation. Shh was overexpressed in adenomas of the colon and exogenous Shh promoted cell proliferation in colonocytes (Douard et al., 2006), whereas Ihh was required for the differentiation of mature colonocytes (van den Brink et al., 2004), suggesting that the regulation of proliferation versus differentiation by Shh and Ihh is widely used during development.

A precise mechanism for the regulation of differentiation by Shh and Ihh binding through the patched (Ptch) receptor has not yet been proven. A previous study, which showed that the Ihh and Shh proteins have similar structures and biological properties (such as a similar affinity for the Hh-binding proteins) but different potencies (Pathi et al., 2001), may provide a basis for the competitive inhibition of Shh. Ihh may behave as a potent competitive inhibitor of Shh for binding to the Ptch receptor, and the relative expression levels of Ihh and Shh may be involved in maintaining a precise balance between the proliferation and differentiation of spinal precursors. However, more direct evidence is needed to reveal the precise mechanism and the roles played by Ihh and Shh in OPC specification.

\section{References}

Blader P, Fischer N, Gradwohl G, Guillemot F, Strähle U (1997) The activity of neurogenin 1 is controlled by local cues in the zebrafish embryo. Development 124:4557-4569. Medline

Chen JK, Taipale J, Cooper MK, Beachy PA (2002) Inhibition of Hedgehog signaling by direct binding of cyclopamine to Smoothened. Genes Dev 16:2743-2748. CrossRef Medline

Chung AY, Kim S, Kim H, Bae YK, Park HC (2011) Microarray screening for genes involved in oligodendrocyte differentiation in the zebrafish CNS. Exp Neurobiol 20:85-91. CrossRef Medline

Currie PD, Ingham PW (1996) Induction of a specific muscle cell type by a hedgehog-like protein in zebrafish. Nature 382:452-455. CrossRef Medline

Douard R, Moutereau S, Pernet P, Chimingqi M, Allory Y, Manivet P, Conti M, Vaubourdolle M, Cugnenc PH, Loric S (2006) Sonic Hedgehogdependent proliferation in a series of patients with colorectal cancer. Surgery 139:665-670. CrossRef Medline

Jung IH, Jung DE, Park YN, Song SY, Park SW (2011) Aberrant Hedgehog ligands induce progressive pancreatic fibrosis by paracrine activation of myofibroblasts and ductular cells in transgenic zebrafish. PLoS One 6:e27941. CrossRef Medline

Jung SH, Kim S, Chung AY, Kim HT, So JH, Ryu J, Park HC, Kim CH (2010) Visualization of myelination in GFP-transgenic zebrafish. Dev Dyn 239: 592-597. CrossRef Medline

Krauss S, Concordet JP, Ingham PW (1993) A functionally conserved homolog of the Drosophila segment polarity gene hh is expressed in tissues with polarizing activity in zebrafish embryos. Cell 75:1431-1444. CrossRef Medline

Kucenas S, Takada N, Park HC, Woodruff E, Broadie K, Appel B (2008) CNS-derived glia ensheath peripheral nerves and mediate motor root development. Nat Neurosci 11:143-151. CrossRef Medline

Kwan KM, Fujimoto E, Grabher C, Mangum BD, Hardy ME, Campbell DS, Parant JM, Yost HJ, Kanki JP, Chien CB (2007) The Tol2kit: a multisite gateway-based construction kit for Tol2 transposon transgenesis constructs. Dev Dyn 236:3088-3099. CrossRef Medline

Lewis KE, Eisen JS (2001) Hedgehog signaling is required for primary motoneuron induction in zebrafish. Development 128:3485-3495. Medline

Li H, de Faria JP, Andrew P, Nitarska J, Richardson WD (2011) Phosphorylation regulates OLIG2 cofactor choice and the motor neuronoligodendrocyte fate switch. Neuron 69:918-929. CrossRef Medline

Park HC, Mehta A, Richardson JS, Appel B (2002) olig2 is required for zebrafish primary motor neuron and oligodendrocyte development. Dev Biol 248:356-368. CrossRef Medline

Park HC, Shin J, Appel B (2004) Spatial and temporal regulation of ventral spinal cord precursor specification by Hedgehog signaling. Development 131:5959-5969. CrossRef Medline

Park HC, Boyce J, Shin J, Appel B (2005) Oligodendrocyte specification in zebrafish requires notch-regulated cyclin-dependent kinase inhibitor function. J Neurosci 25:6836-6844. CrossRef Medline

Pathi S, Pagan-Westphal S, Baker DP, Garber EA, Rayhorn P, Bumcrot D, Tabin CJ, Blake Pepinsky R, Williams KP (2001) Comparative biological responses to human Sonic, Indian, and Desert hedgehog. Mech Dev 106:107-117. CrossRef Medline

Poncet C, Soula C, Trousse F, Kan P, Hirsinger E, Pourquié O, Duprat AM, Cochard P (1996) Induction of oligodendrocyte progenitors in the trunk neural tube by ventralizing signals: effects of notochord and floor plate grafts, and of sonic hedgehog. Mech Dev 60:13-32. CrossRef Medline

Pringle NP, Yu WP, Guthrie S, Roelink H, Lumsden A, Peterson AC, Richardson WD (1996) Determination of neuroepithelial cell fate: induction of the oligodendrocyte lineage by ventral midline cells and sonic hedgehog. Dev Biol 177:30-42. CrossRef Medline

Rowitch DH (2004) Glial specification in the vertebrate neural tube. Nat Rev Neurosci 5:409-419. CrossRef Medline

Scheer N, Campos-Ortega JA (1999) Use of the Gal4-UAS technique for targeted gene expression in the zebrafish. Mech Dev 80:153-158. CrossRef Medline

Shin J, Park HC, Topczewska JM, Mawdsley DJ, Appel B (2003) Neural cell fate analysis in zebrafish using olig2 BAC transgenics. Methods Cell Sci 25:7-14. CrossRef Medline

Sun Y, Meijer DH, Alberta JA, Mehta S, Kane MF, Tien AC, Fu H, Petryniak MA, Potter GB, Liu Z, Powers JF, Runquist IS, Rowitch DH, Stiles CD 
(2011) Phosphorylation state of Olig2 regulates proliferation of neural progenitors. Neuron 69:906-917. CrossRef Medline

van den Brink GR, Bleuming SA, Hardwick JC, Schepman BL, Offerhaus GJ, Keller JJ, Nielsen C, Gaffield W, van Deventer SJ, Roberts DJ, Peppelen- bosch MP (2004) Indian Hedgehog is an antagonist of Wnt signaling in colonic epithelial cell differentiation. Nat Genet 36:277-282. CrossRef Medline 